\title{
NMR-Relaxometer for Diagnosis and Control of Chronic Kidney Disease Patients Parameters (Urea and Creatinine)
}

\author{
Rustem Sultan-Hamit Kashaev, Liliana Rashidovna Khaziahmetova \\ Kazan State Power Engineering University, Kazan, Republic Tatarstan, Russian Federation \\ Email: kashaev2007@yandex.ru
}

Received 19 October 2014; revised 28 November 2014; accepted 17 December 2014

Copyright (C) 2014 by authors and OALib.

This work is licensed under the Creative Commons Attribution International License (CC BY). http://creativecommons.org/licenses/by/4.0/

(c) †) Open Access

\begin{abstract}
Determination of disease stages and control of changes in blood and plasma during kidney injury (KJ) cure can be done using nuclear (proton) magnetic resonance (NMR) parameters dependences from physical-chemical parameters of blood changes. NMR spectroscopy allows the monitoring of molecular recognition processes in solution. For realization of this approach, the low field NMR relaxometer was elaborated. Measurements of relaxation parameters were performed. Dependences of relaxation times T2A from urea and serum creatinine were received.
\end{abstract}

\section{Keywords}

Acute, Kidney, Injury, Nuclear, Magnetic, Resonance

Subject Areas: Biochemistry, Biophysics

Acute kidney injury (AKI) is one of the popular topics of discussions due to increasing development of spectroscopic methods recently. The disease progression and prognosis may be determined by this method in blood and urine specimens. Since AKI is associated with disease conditions, prevention and early detection of AKI becomes important in clinical settings. Early detection of AKI and its subsequent resolution using spectroscopy parameters could predict subsequent development of intrinsic AKI, dialysis requirement, duration of intensive care and finally affect mortality.

Chertow and colleques noticed that a rise in serum creatinine (SCr) of just $\geq 0.3 \mathrm{mg} / \mathrm{dl}$ had a four-fold higher multivariable-adjusted risk of death [1]. The AKI Network (AKIN) group modified the AKI definition based on RIFLE classification [2] classified the patients with the change in SCr $\geq 0.3 \mathrm{mg} / \mathrm{dl}(\geq 26.4 \mu \mathrm{mol} / \mathrm{l})$ within 48 
hours as AKIN stage I, whereas patients receiving renal replacement therapy are included in AKIN stage 3.

Nuclear magnetic resonance (NMR), especially MR-tomography has become a familiar term in medical community. This is due to the advance and powerfulness of NMR-methods. But there exists some hesitations to examine by this method high risk patients (e.g. under sedation or anaestesia, stroke etc.), because powerful magnet fields affects the general state of patients.

Contemporary diagnosis is oriented on the non-invasive and nondestructive forms. Medical ordinary clinics need cheap devices for early identification and determination of disease stages and control of their cure stages and changes in blood physchemical parameters during kidney disease cure.

It can be done using nuclear (proton) magnetic resonance (NMR) parameters dependences from physicalchemical characteristics of blood changes. NMR spectroscopy allows the monitoring of molecular recognition processes in solution. Nowadays, a plethora of NMR methods are available to deduce the key features of the interactions in tissues and blood components [3]. To realize such approach the suitable device is to be elaborated and corresponding NMR relations between NMR-spectroscopic parameters and blood characteristics must be revealed.

We constructed and proposed portable low field NMR-relaxometer, presented at Figure 1.

Its power supply is autonomous-from accumulator or grid. Control and data processing is performed by Notebook. Portable magnetic system is constructed from NdFeB alloys with magnetic field $B_{0}=0.242 \mathrm{Tl}$. Inhomogeneity of $B_{1}<2 \%$ in $75 \%$ of its coil volume. Coefficient of sensitivity is $K=v_{0}^{2} \cdot D^{3}\left[\mathrm{MHz}^{2} \mathrm{~cm}^{3}\right]=2700-$ 4150, where proton resonance frequency $v_{0}=10-12 \mathrm{MHz}, D=10-30 \mathrm{~mm}$-diameter of the probe head coil. Consumed power $P<15$ VA. Mass $<15 \mathrm{~kg}$. Error of NMR-parameters measurements $\pm 4 \%$ [4] [5].

Measurements of relaxation parameters were performed by Carr-Purcell-Meiboom-Gill-method [6] using $90^{\circ}-\tau-\left(180^{\circ}-2 \tau \text { - }\right)_{\mathrm{N}}$ pulse sequence, where $N$-number of $180^{\circ}$-pulses. Two spin-spin relaxation times $T_{2 \mathrm{~A}}, T_{2 \mathrm{~B}}$ with corresponding proton populations $P_{2 \mathrm{~A}}, P_{2 \mathrm{~B}}$ corresponding to water and albumin were observed. For $T_{2 \mathrm{~A}}$ we used measurement parameters: period of sequences $T=6 \mathrm{~s}$, interval between pulses $\tau=200-400 \mu \mathrm{s}$, number of pulses $N=5000$, number of accumulations $n=3$. For $T_{2 \mathrm{~B}}$ : $T=200 \mathrm{~ms}, \tau=200 \mu \mathrm{s}, N=100, n=50-100$ in the regime of water phase saturation.

Previously at resonance frequency $v_{0}=100 \mathrm{MHz}$ using NMR spectrometer Tesla-BS 100 we obtained NMRspectra of blood plasma in the frequency range $v=99-100 \mathrm{MHz}$, presented at Figure 2.

In the range of chemical shifts $\delta_{1}=v_{1} / v_{0} \approx-1-2.5 \mathrm{ppm}$ (in the right side of spectra) was observed a series of peaks with common intensity $38.5 \%$ (integral intensity of the whole spectra is $100 \%$ ), corresponding to -CHO, $-\mathrm{CH}_{2}$ and $-\mathrm{CH}_{3}$ groups of albumen molecules. At $\delta_{2}=v_{2} / v_{0} \approx 4-6 \mathrm{ppm}$ appear series of highly resolved peaks (with common intensity $47.2 \%$ ), corresponding to water molecules at different states in blood plasma. Line widths $\Delta \delta$ of albumen peaks are $\Delta \delta \approx 2-5 \mathrm{~Hz}$. For water peak sit is $\Delta \delta \approx 1 \mathrm{~Hz}$. Calculations of corresponding spin-spin relaxation times from the line widths were made using equation:

$$
\left(T_{2}\right)_{\mathrm{obs}}^{-1}=\left(T_{2}^{*}\right)^{-1}+\pi \Delta \delta
$$

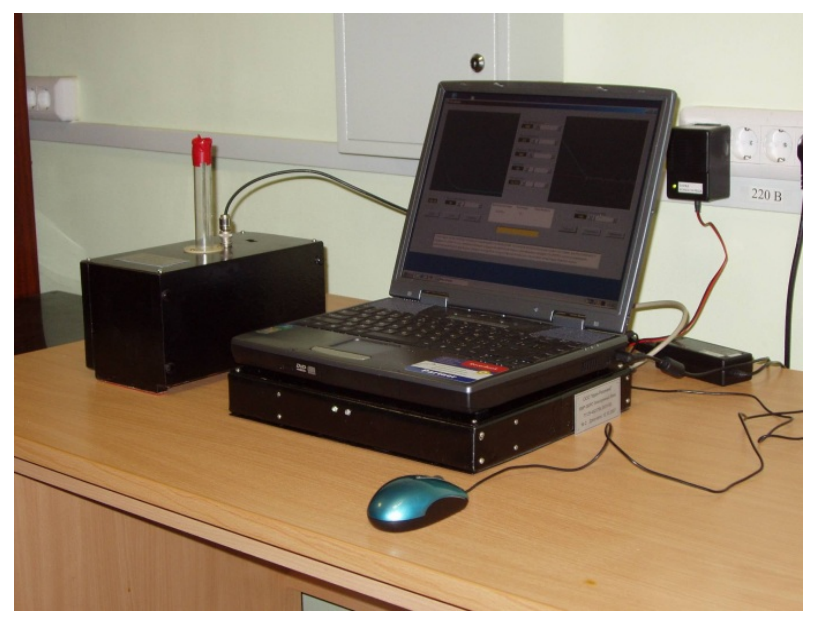

Figure 1. Portable low field NMR-relaxometer. 
where $T_{2 \mathrm{obs}}$-observed relaxation time, $T_{2}^{*}$-relaxation time, caused by magnetic field in homogeneity. Considering that for water $\Delta \delta \approx 1 \mathrm{~Hz}$ and $\left(T_{2}^{*}\right)^{-1} \approx \pi \Delta \delta^{*}=3.14 \times 10^{-6}$, from spectra at Figure 2 we from Equation (1) obtain $T_{2} \approx 300-400$ ms relaxation times of blood plasma. For albumen they are expected to be $T_{2 \mathrm{~B}} \approx 30-40$ mc with protons population $P_{2 \mathrm{~B}} \approx 14 \%$.

Really, from relaxation measurements using Carr-Purcell-Meiboom-Gill-method [6] it was established, that in plasma relaxation times $T_{2 \mathrm{~A}}$ and $T_{2 \mathrm{~B}}$ (see Table 1 ), values are near to calculated from spectra.

First column designate No. of patient, year of birth, before or after hemodialysis (HD), the second and third columns-measured $T_{2 \mathrm{~A}}$ and $T_{2 \mathrm{~B}}$, fourth column $-P_{2 \mathrm{~A}, \mathrm{~B}}$, fifth and sixth columns—changes of relaxation times.

Values of Urea (mol. weight $=60$ a.u.) and serum Creatinine (m.w. $=113$ a.u.), one the important parameters of kidney decease were measured in standard biochemical laboratory of Kazan State Medical Academy using LAHEMA set and Popper method. Obtained NMR-parameters dependences of corrected $T_{2 \mathrm{~A}} / \mathrm{Alb}$ for albumen weight are presented at Figure 3 and Figure 4.

Reserved dependences can be approximated with 95\% confidence interval by relations:

$$
\begin{gathered}
U=38.1-0.016 \cdot T_{2 \mathrm{~A}} / \mathrm{Alb} \\
C=1.61 \exp \left(-T_{2 \mathrm{~A}} / 1257 \cdot \mathrm{Alb}\right)
\end{gathered}
$$

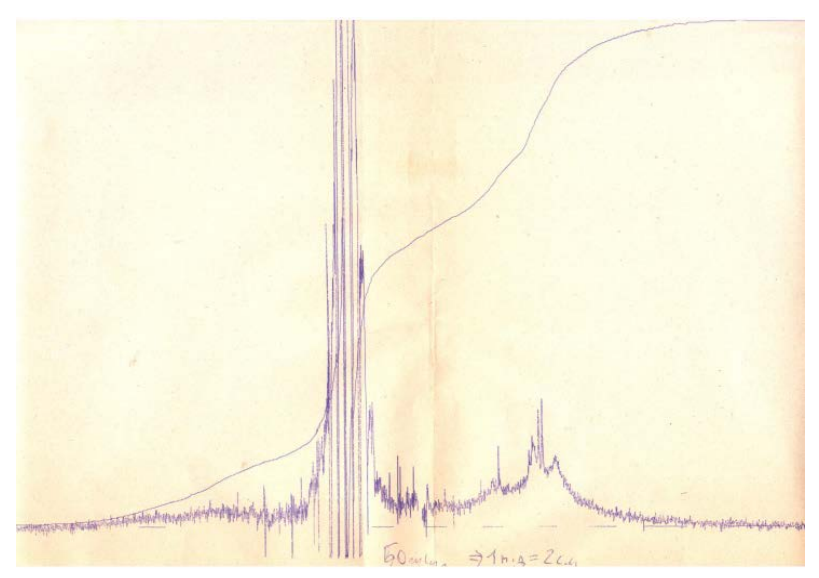

Figure 2. NMR-spectra of blood plasma.

\begin{tabular}{|c|c|c|c|c|c|}
\hline No. patient/year of birth (y.b.) & $T_{2 \mathrm{~A}}(\mathrm{~ms})$ & $T_{2 \mathrm{~B}}(\mathrm{~ms})$ & $P_{\mathrm{A}}(\%)$ & $\Delta T_{2 \mathrm{~A}} / \%$ & $\Delta T_{2 \mathrm{~B}} / \%$ \\
\hline \multicolumn{6}{|c|}{ Patient of chronic kidney disease } \\
\hline No. 1, 1950 y.b., before HD & $667 \pm 22$ & $404 \pm 70$ & $95 \pm 3$ & - & - \\
\hline No. 1, after HD & $519 \pm 16$ & $256 \pm 42$ & $94 \pm 2$ & $-148 /-28.5$ & $-148 /-57.8$ \\
\hline No. 1 , after 1.5 years, before HD & $529 \pm 23$ & & & & \\
\hline No. 1 , after 1.5 years, after HD & $410 \pm 4$ & & & $-119 /-29$ & \\
\hline No. 2, 1941 y.b., before HD & $671 \pm 2$ & $374 \pm 25$ & $97 \pm 1$ & - & - \\
\hline No. 2, after HD & $577 \pm 3$ & $338 \pm 70$ & $97 \pm 3$ & $-94 /-16.3$ & $-6 /-10.6$ \\
\hline No. 3, 1942 y.b., before HD & $468 \pm 19$ & $230 \pm 26$ & $89 \pm 9$ & - & - \\
\hline \multirow[t]{2}{*}{ No. 3, after HD } & $377 \pm 9$ & $182 \pm 30$ & $89 \pm 6$ & $-91 /-24.1$ & $-48 /-26.4$ \\
\hline & \multicolumn{3}{|c|}{ Healthy patients } & & \\
\hline No. 1, 1980 y.b. & $594 \pm 14$ & $284 \pm 22$ & $85 \pm 3$ & - & - \\
\hline No. 1, 1981 y.b. & $575 \pm 11$ & $293 \pm 20$ & $88 \pm 4$ & - & - \\
\hline
\end{tabular}

Table 1. Relaxation times and there changes for different stages of chronic kidney decease. 


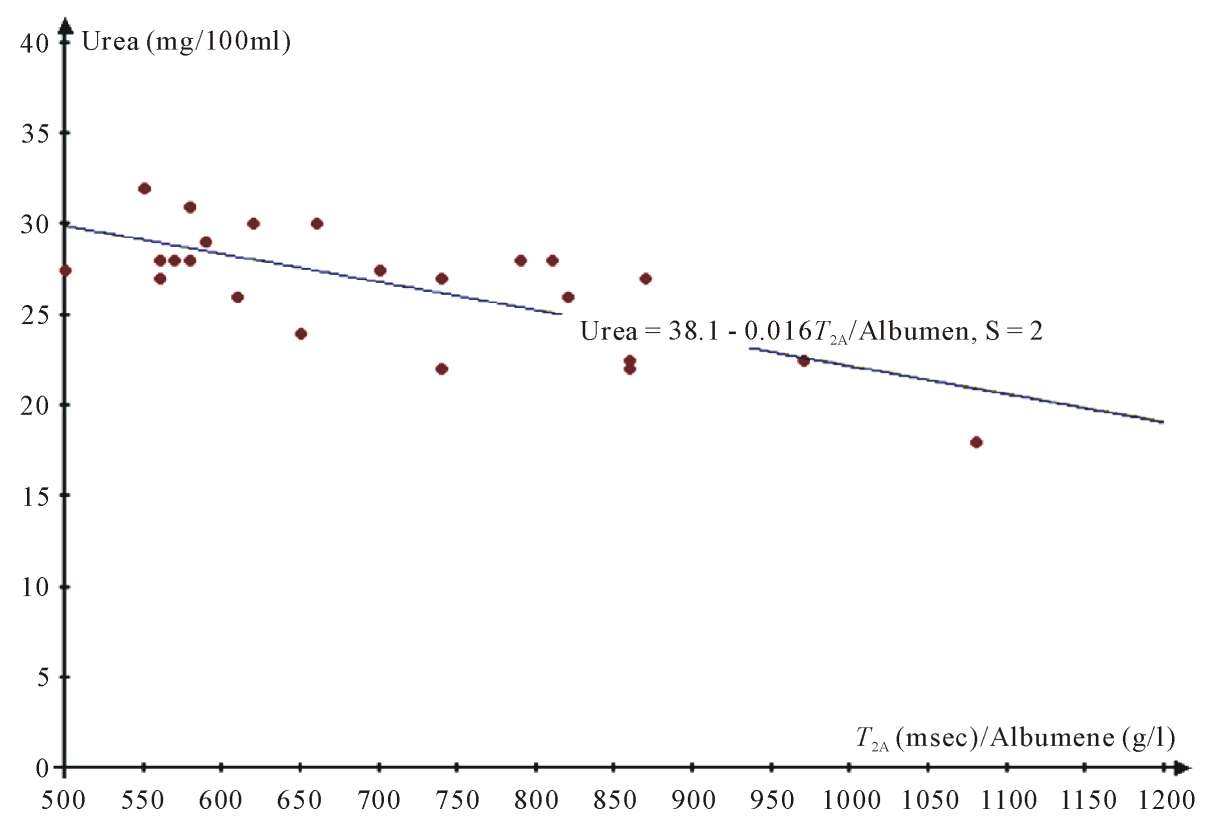

Figure 3. Dependence of Urea from $T_{2 \mathrm{~A}} /$ Alb.

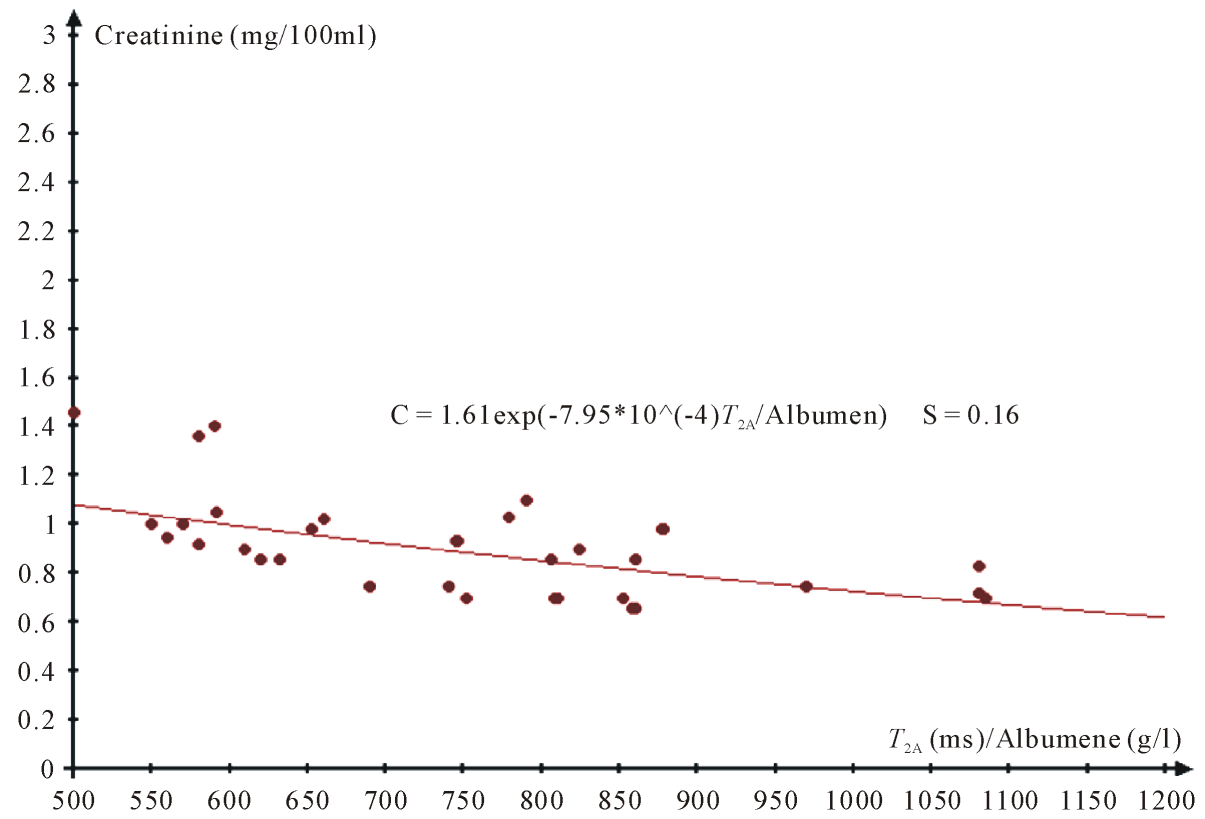

Figure 4. Creatinine from $T_{2 \mathrm{~A}} /$ Alb.

Standard deviation for urea and creatinine are 2.2 and 0.16 ( $\pm 7.3 \%$ and $\pm 14.6 \%)$.

Obtained results can be explained on the base of increase of substances with middle molecular weight (acting as endotoxines) at pathology, leading to decrease of $T_{2 \mathrm{~A}}$.

At Figure 5 presented dependence of $T_{2 \mathrm{~A}}$ from age $t$ (years) of healthy men, which with the error 20 MS ( 10\%) can be approximated by linear equation:

$$
T_{2 \mathrm{~A}}=0.8 t+141
$$

So, age must be considered at Urea and Creatinine determination. It should be also mentioned, that respiratory diseases (tonsillitis, grippe and headache) increase relaxation times on 15\% - 30\% from their average values, for 


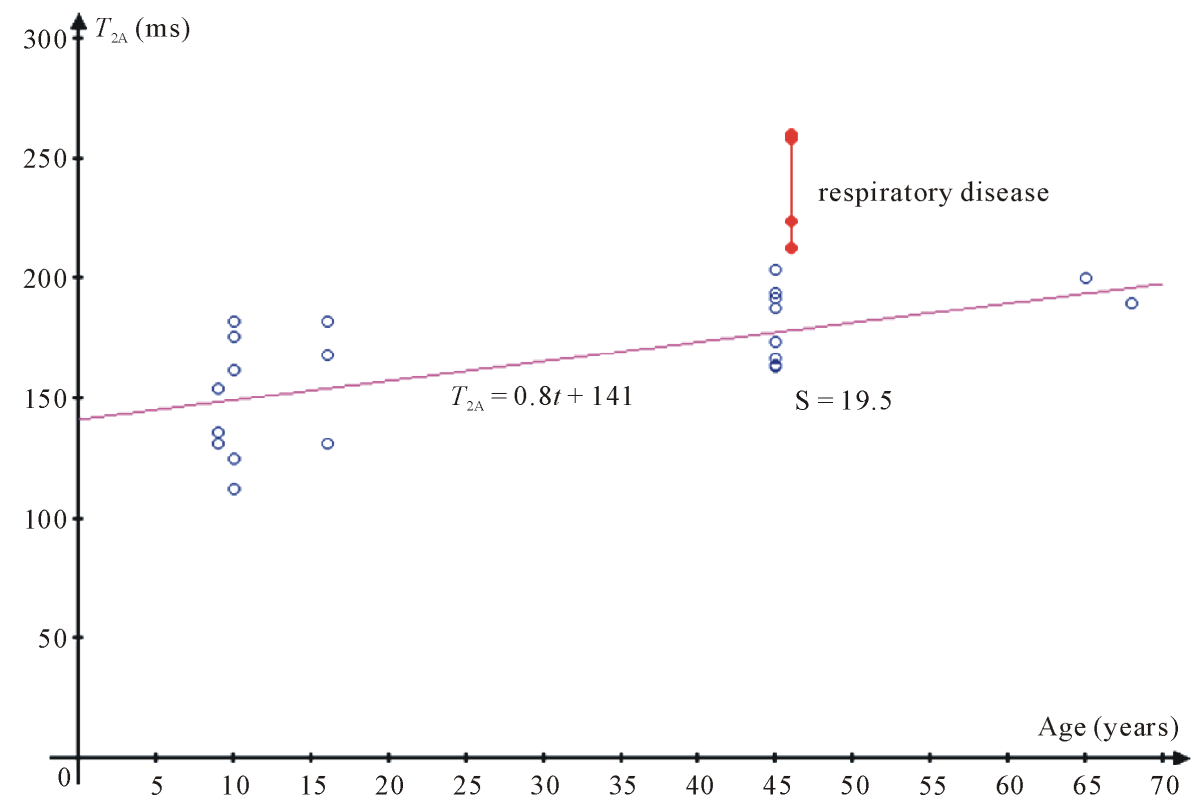

Figure 5. Dependence of $T_{2 \mathrm{~A}}$ from age of healthy men.

instance from $T_{2 \mathrm{~A}}=180 \mathrm{MS}$ to $T_{2 \mathrm{~A}}=213-260 \mathrm{MS}$ of 45 years old patient. At recover $T_{2 \mathrm{~A}}$ return to its individual norm.

Received results show, that NMR-relaxation method can be used for express-analysis of chronic kidney disease parameters before and after hemodialysis and characterizing different stages of AKI.

\section{References}

[1] Chertow, G.M., Burdick, E., Honour, M., Bonventre, J.V. and Bates, D.W. (2005) Acute Kidney Injury, Mortality, Length of Stay, and Costs in Hospital Patients. Journal of the American Society of Nephrology, 16, 3365-3370. http://dx.doi.org/10.1681/ASN.2004090740

[2] Bellomo, R., Ronco, C., Kellum, J.A., Mehta, R.L. and Palevsky, P. (2004) Acute Renal Failure—Definition, Outcome Measures, Animal Models, Fluid Therapy and Information Technology Needs: The Second International Conference of the Acute Dialysis Quality Initiative (ADQI) Group. Critical Care, 8, R204-R212. http://dx.doi.org/10.1186/cc2872

[3] Unione, L., Galante, S., Diaz, D, Canada, F.J. and Barbero, J.J. (2014) NMR and Molecular Recognition. The Application of Ligand-Based NMR-Methods to Monitor Molecular Interactions. Medicinal Chemistry Communications, 5, 1280-1289. http://dx.doi.org/10.1039/C4MD00138A

[4] Kashaev, R.S., Idiatullin, Z.Sh. and Temnikov, A.N. (2007) Portable Relaxometer NMR. RF Patent No. 67719, Bulletins of Inventions No. 30.

[5] Kashaev, R.S., Idiatullin, Z.Sh. and Temnikov, A.N. (2008) Device for Thermostating of Sample in the NMR Probehead. Patent of RF No. 2319138, Bulletins of Inventions No. 32.

[6] Meiboom, S. and Gill, D. (1958) Modified Spin-Echo Method for Measuring Nuclear Relaxation Times. Review of Scientific Instruments, 29, 688-691. 